\title{
GENESIS OF A PHU DISASTER RESPONSE PLAN
}

A t 10.27 am on December 28,1989 , Newcastle was hit by an earthquake measuring 5.6 on the openended Richter scale. Twelve people were killed by collapsed buildings and 105 people presented to Hunter Hospitals with symptoms directly attributable to the earthquake.

Although the toll from the Newcastle earthquake was relatively small when compared with overseas earthquakes, the possibility of massive loss of life focused attention on the Health Department's counterdisaster medical contingency plans. A disaster medical aid system must respond quickly and appropriately, utilising the most relevant organisational concepts and the most advanced medical procedures available.

Since medical knowledge and especially medical technology are developing at an exponential rate $e^{2}$ counter-disaster medical plans must be continually revised. For more than a decade each contingency plan developed by the NSW Health Department has incorporated a mechanism to ensure that the plan is continually renewed.

\section{NSW disaster response plans}

From the mid 1970s the revised Sydney Metropolitan Disaster Plan was kept under regular review. As a result its organisational structure was recast in the NSW Disaster Medical Plan promulgated in 1985. This latter was established as a master plan on which regional plans were to be based. Again the new structure emphasised that "Plans at all levels must be subject to regular review, so that an increasingly effective overall plan may evolve to facilitate response to any disaster situation which may occur in any part of this State"3.

In July 1990 Medplan, designed to be compatible with the NSW Disaster Plan (NSW Displan), was released as a new model set of procedures. All Area and Regional Health Services and all ambulance districts and regions were required to maintain disaster plans which were operationally compatible with Medplan.

This document identifies in specific detail what review procedures are to be undertaken and who has executive responsibility for each. For example, a post-incident debrief of all Health Department agencies must be held and chaired by a person who did not participate in the disaster response. The aim of such debrief is to generate information to assist in assessing the efficiency of Medplan procedures and the adequacy of human and material resources ${ }^{4}$ and to offer psychological support for staff in the post-disaster period.

The debriefing provisions of Medplan established a procedure to help make effective the Health Department's 1985 recommendation designed to ensure some system renewal. These provisions also reflected the Department's determination to learn from experience. Because disasters of any kind are relatively rare, it is imperative that the Department take advantage of the few occasions when its contingency plans are, of necessity, realistically field-tested.

\section{Hunter Area disaster response plans}

The Hunter Area Health Service (HAHS) was created in August 1988 by amalgamating the four health services ff the Greater Newcastle, Lower Hunter, Northumberland and Upper Hunter areas. Authority for the organisation of a local medical response to a disaster was then vested in the HAHS. In the early months of the new body's existence, time and energy were spent establishing administrative and professional structures. Thus it was not possible to revise local medical disaster plans to meet the needs of the new area before the unexpected earthquake occurred.

The consequence of these chance events was that local health professionals generally had to assume the roles and protocols established by the NSW Disaster Medical Plan of 1985. This process generated a medical response which was relatively efficient, but also highlighted the need to revise existing plans.

In 1990 and 1991 the local disaster medical response plan pertaining to the HAHS was written, incorporating some suggestions arising from the experience of the earthquake. This plan has been named the Hunter Multiple Casualty, Emergency and Disaster Medical Response Plan (Hunter Medplan).

During the preparation of Hunter Medplan, it was recognised that previous disaster plans did not include public health response. PHUs, which were not established until $1990^{5}$, had no designated roles in counter-disaster activities. Moreover PHU and other personnel have had little opportunity to complete the detailed identification of such roles. The earthquake in Newcastle focused attention on the need to create a clear and workable counter-disaster plan, complementary to the Hunter Medplan, for the PHU.

\section{The Hunter Public Health Unit}

The Hunter PHU was established in March 1990 to evaluate and coordinate local health priorities and to develop associated public health strategies relating to infectious diseases, environmental health, reproductive health, chronic diseases and injuries.

Environmental Health Officers were formally integrated into the PHUs in August 1991, and were also allotted additional duties under the Medical Officer of Health and Director of the PHU.

\section{THE PUBLIC HEALTH UNIT DISASTER RESPONSE PLAN}

The PHU has a vital role in implementing appropriate responses to disasters where the health of the public is threatened. The geographic and demographic profile of the Hunter Area dictates the kind of disasters which may occur ${ }^{6}$ and includes natural disasters such as earthquakes, epidemics, fires and floods and humancaused disasters such as chemical spills, industrial accidents and major transport accidents.

The aims of the PHU in a disaster are:

- to coordinate and cooperate with other agencies and to protect and maintain the health of the public; and

- to ensure the most efficient and effective use of public health resources. 


\section{Genesis of a disaster plan}

\section{Continued from page 73}

The Hunter PHU disaster response plan outlines the possible effects of disasters, both on the environment and on the health of the public, and then outlines recommended responses to these possibilities. The roles of all PHU staff members are clearly delineated with a specific chain of command (Figure 1), and important links with other counter-disaster personnel described.

\section{Comprehensive reference section}

Guidelines to help a staff member implement a PHU response to diverse disasters constitutes an important section of the plan.

It was recognised that a PHU disaster response plan needed to be clear and easy to read and implement. With this in mind, the Hunter PHU plan was designed so individual sections could be summarised into concise action plans. Another important section in any disaster plan is the list of resource people and data bases. This list identifies, for example, all the significant community leaders and experts who can be called on in an emergency, with telephone or other contact information. Experience with previous counter-disaster plans indicates this list must be scrupulously accurate, and updated at regular intervals. To this end, responsibility for list revision must be vested in one person with the expectation that the lists will be checked at least twice a year.

\section{Relationship with other disaster response plans}

The Hunter PHU disaster response plan was designed to be an integral part of Hunter Medplan, which in turn was designed to complement NSW Medplan. NSW Medplan is operationally compatible with NSW Displan. The schematic illustration of the four plans can be seen in Figure 2.

Copies of the Hunter PHU Disaster Response Plan can be obtained from Jeanette Sheridan, Hunter Area Health Service PHU, PO Box 11A, Newcastle 2300. Telephone (049) 291 292; facsimile (049) 294037.

Thais A Miles, Public Health Officer, NSW Health Department (seconded to the Public Health Unit, Hunter Area Health Service) Jeanette Sheridan, Planning Officer, Public Health Unit, Hunter Area Health Service

1. Mahoney LE, Reutershan TP. Catastrophic disasters and the design of disaster medical care systems. Ann Emerg Med 1987; 16:1085-1091. 2. Callahan D. Shattuck Lecture - Contemporary biomedical ethics. N Engl J Med 1980; 302:1228-1233.

3. Department of Health, New South Wales. New South Wales Disaster Medical Plan. Sydney. NSW Health Department, 1985.

4. Department of Health, New South Wales. New South Wales multiple casualty emergency and disaster medical response plan (Medplan). Sydney. NSW Health Department, 1990.

5. Rubin G, Frommer M, Morey S, Leeder S. On the right track.

Public Health Bulletin 1991; 2(1):1-2.

6. Sheridan J, Miles T, Mitchell M. Hunter Area Health Service Public Health Unit Disaster Response Plan. Newcastle. Hunter Area Health Service, 1991.

\section{FIGURE 1}

HUNTER PUBLIC HEALTH UNIT CHAIN OF COMMAND IN A DISASTER

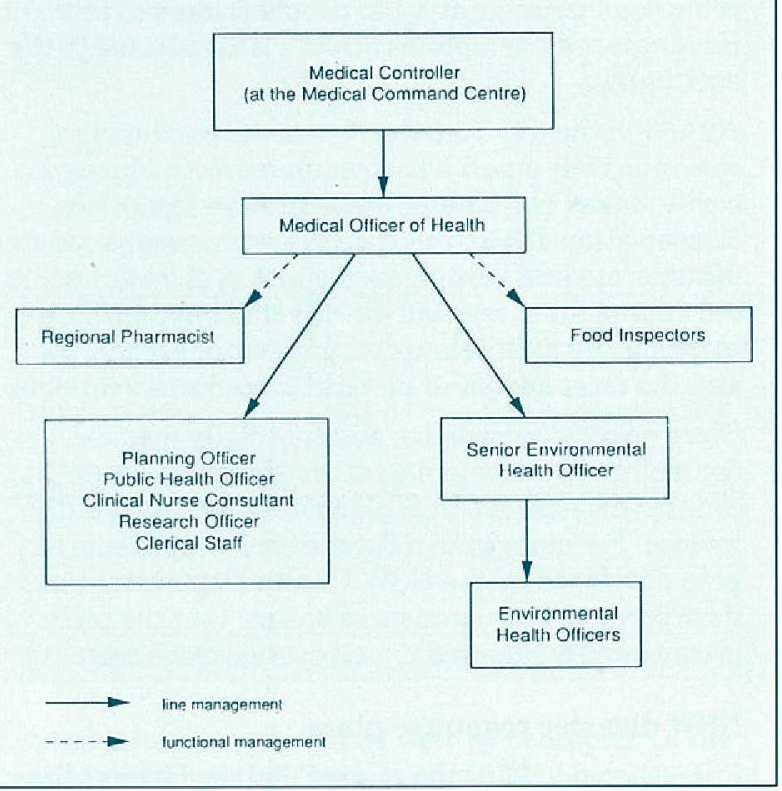

FIGURE 2

SCHEMATIC ILLUSTRATION OF THE RELATIONSHIP BETWEEN FOUR DISASTER RESPONSE PLANS

NSW Displan 